\title{
Publisher Correction: Long spin diffusion lengths in doped conjugated polymers due to enhanced exchange coupling
}

Shu-Jen Wang, Deepak Venkateshvaran (D, M. R. Mahani, Uday Chopra, Erik R. McNellis, Riccardo Di Pietro, Sam Schott D, Angela Wittmann, Guillaume Schweicher (D), Murat Cubukcu, Keehoon Kang, Remington Carey (D),

Thomas J. Wagner, Janis N. M. Siebrecht, Daniel P. G. H. Wong, lan E. Jacobs (D, Razan O. Aboljadayel, Adrian lonescu, Sergei A. Egorov, Sebastian Mueller, Olga Zadvorna, Piotr Skalski, Cameron Jellett, Mark Little, Adam Marks, lain McCulloch, Joerg Wunderlich, Jairo Sinova and Henning Sirringhaus

Correction to: Nature Electronics https://doi.org/10.1038/s41928-019-0222-5, published online 18 March 2019.

Owing to a technical error, the 'Published online' date of this Article originally mistakenly appeared as '15 March 2019 ', but should have been '18 March 2019'. 